\title{
A Modified Natural BEM for Exterior Problems of the Helmholtz Equation Quan Zheng ${ }^{1, a}$, Yue Gao ${ }^{1, b}$ \\ ${ }^{1}$ College of Sciences, North China University of Technology, Beijing 100144, China \\ azhengq@ncut.edu.cn,b846888286@qq.com
}

Keywords: Helmholtz equation on infinite regions; modified DtN operator; natural boundary element method; existence and uniqueness of solution; error estimate

\begin{abstract}
In this paper, a modified natural boundary element method (MNBEM) by using a modified DtN operator to improve the natural boundary element method (NBEM) is proposed for Neumann BVPs of the Helmholtz equation on unbounded domains. We prove the existence and the uniqueness of the solution of its variational problem in $L^{2}(\Gamma)$, and obtain $L^{2}$ error estimate of the boundary element solution which depends on the wave number $k$, the radius $R$ of the boundary, the exact solution $u$, the mesh size $h$ and the number $N$ of truncated terms taken from the infinite series. The numerical results confirm the well-posedness and the convergence.
\end{abstract}

\section{Introduction}

Natural boundary integral equations for exterior Neumann BVPs can be solved directly by using the finite element method. That is the natural boundary element method (NBEM). K. Feng and D. Yu established the natural boundary integral equations by Green's functions on typical unbounded domains, then obtained boundary element solutions, and also applied the natural boundary integral equations in the coupling method and the domain decomposition method for the exterior problems [1-2]. R. Li obtained stiffness matrix of the NBEM corresponded to the linear element and the quadratic element for the exterior Helmholtz problem, and deduced error estimates [3]. Although sequences of absorbing boundary conditions with increasing accuracy and modified DtN (MDtN) boundary conditions were introduced into artificial boundary condition methods [4-6], and the error estimates depending on $N, R$ and $h$ were derived for the DtN-FEM coupling [7-9], the MDtN operator should be introduced into the NBEM for the exterior Helmholtz problem.

In this paper, a MNBEM for exterior Neumann BVPs of the Helmholtz equation is studied. We introduce the DtN operator and the MDtN operator in section 2; propose the MNBEM using a modified DtN operator, prove the uniqueness of solution of the variational problem and deduce the error estimate in section 3; present numerical examples in section 4; and finally make conclusions.

\section{From a DtN operator to an MDtN operator}

We consider the exterior Helmholtz problem

$$
\begin{aligned}
& \Delta u+k^{2} u=0, \quad \text { in } \Omega, \\
& \frac{\partial u}{\partial n}=g, \quad \text { on } \quad \Gamma, \\
& \frac{\partial u}{\partial r}-i k u=o\left(r^{-\frac{1}{2}}\right), \quad r \rightarrow \infty,
\end{aligned}
$$

where $\Gamma$ is a circle of radius $R$ whose center is the origin of coordinates, $\Omega$ is an unbounded domain in the plane $R^{2}$ with boundary $\Gamma, n$ is the outer normal to $\Gamma$ and wave number $k>0$.

The natural boundary integral equation of (1) (2) is

$$
\frac{\partial u}{\partial n}=K u(\theta), \quad \text { on } \quad \Gamma,
$$

which is called a DtN operator, where $u(\theta)=u(R, \theta)$ and $K$ is the boundary integral operator: 


$$
\begin{aligned}
& K u(\theta)=\frac{k}{2 \pi} \int_{0}^{2 \pi}\left[\sum_{m=-\infty}^{+\infty} H_{|m|}(k R) \cos m\left(\theta-\theta^{\prime}\right) u\left(\theta^{\prime}\right) d \theta^{\prime}\right], \\
& H_{m}(z)=-\frac{\frac{d H_{m}^{(1)}(z)}{d z}}{H_{m}^{(1)}(z)}=\frac{H_{m+1}^{(1)}(z)}{H_{m}^{(1)}(z)}-\frac{m}{z}, \quad m=0,1, \cdots,
\end{aligned}
$$

where $H_{m}^{(1)}(z)$ is the Hankel function of the first kind of order $m$.

Let $g^{\in} L^{2}(\Gamma)$, then the variational problem corresponding to (4) is: Find $u^{\in} H^{1}(\Gamma)$ such that $a(u, v)=<g, v>$,

where $a(u, v)=\langle K u, v\rangle,\langle g, v\rangle=\int_{\Gamma} g \bar{v} d s$.

Lemma 1. For all $z>0$ and for all $m, m^{\prime} \in R$ satisfying $|m|>\left|m^{\prime}\right|$, we have

$$
\operatorname{Im}\left(-H_{\left|m^{\prime}\right|}(z)\right)>\operatorname{Im}\left(-H_{|m|}(z)\right)=\frac{2}{\pi z\left|H_{|m|}^{(1)}(z)\right|^{2}}>0 \text { and } \operatorname{Re}\left(H_{|m|}(z)\right)>0 .
$$

Lemma 2 The linear operator $K: H^{s}(\Gamma) \rightarrow H^{s-1}(\Gamma)$ defined by (4) satisfies

$$
\sqrt{\frac{2 \pi}{R} c_{1}}\|w\|_{s, \Gamma} \leq\|K w\|_{s-1, \Gamma_{0}} \leq \sqrt{\frac{2 \pi}{R} c_{2}}\|w\|_{s, \Gamma},
$$

where $s$ is any real number, $c_{1}=\inf _{m \in N}\left|\frac{k R H_{|m|}(k R)}{\sqrt{1+m^{2}}}\right|^{2}>0$ and $c_{2}=\sup _{m \in N}\left|\frac{k R H_{|m|}(k R)}{\sqrt{1+m^{2}}}\right|^{2}$.

Theorem1. Let $g^{\in} L^{2}(\Gamma)$, then the variational problem (7) has a unique solution $u^{\in} H^{1}(\Gamma)$.

The problem (7) can be truncated into the following variational problem: Find $u^{N \in} H^{1}(\Gamma)$ s.t.

$$
a^{N}\left(u^{N}, v\right)=<g, v>\text {. }
$$

In fact, the truncation imposes an incorrect boundary condition $\frac{\partial u}{\partial n}=0$ for the modes with $|m|>N$. To avoid the problems caused by truncation, Grote and Keller proposed the modified DtN boundary condition (see [5]).

According to the ideas, in this paper, we take

$$
\frac{\partial u}{\partial n}=B u \equiv-\left(i k-\frac{1}{2 R}\right) u, \quad \text { on } \Gamma,
$$

the MDtN operator is defined by:

$$
\frac{\partial u}{\partial n}=L^{N} u \equiv K^{N} u-\varsigma \sum_{|m| \leq N} u_{m} e^{i m \theta}+\varsigma u, \quad \text { on } \Gamma
$$

where $\varsigma=-i k+\frac{1}{2 R}, u_{m}$ is the Fourier coeffcient of $u(\theta)$, i.e. $u_{m}=\int_{0}^{2 \pi} u(\theta) d \theta$.

We can see that $B$ only replaces the action of $K$ on the higher modes $(|m|>N)$, without modifying the action of $K$ on the lower modes. And by Lemma 1, the imaginary parts of $H_{|m|}(k R)$ and $\zeta$ are both negative, and the real parts of and $H_{|m|}(k R)$ and $\zeta$ are both positive.

\section{A modified NBEM and its error estimate}

Let $g^{\in} L^{2}(\Gamma)$, then the variational problem corresponding to (7) is: Find $u^{N \in} H^{1}(\Gamma)$ such that $b^{N}\left(u^{N}, v\right)=<g, v>$

where $b^{N}(u, v)=\left\langle L^{N} u, v>,\langle g, v\rangle=\int_{\Gamma} g \bar{v} \mathrm{~d} s\right.$.

Lemma 3. The linear operator $L^{N}: H^{s}(\Gamma) \rightarrow H^{s-1}(\Gamma)$ satisfies

$$
\left\|L^{N} w\right\|_{H^{s-1}(\Gamma)} \leq c_{3}\|w\|_{H^{s}(\Gamma)} \text { and }\left\|L^{N} w\right\|_{L^{2}(\Gamma)} \leq c_{3}\|w\|_{L^{2}(\Gamma)},
$$

where $C_{3}=\sqrt{2 \pi R \max \left\{\max _{|m| \leq N}\left|\frac{k H_{|m|}(k R)}{\sqrt{1+m^{2}}}\right|^{2}, \frac{|s|^{2}}{1+(1+N)^{2}}\right\}}, C_{4}=\sqrt{2 \pi R \min \left\{\min _{|m| \leq N}\left|k H_{|m|}(k R)\right|^{2},|s|^{2}\right\}}$ and $s$ is any real number.

Theorem 2. Let $g^{\in} L^{2}(\Gamma)$, then the variational problem (12) has a unique solution $u^{N \in} L^{2}(\Gamma)$.

Lemma 4. $\operatorname{Im}\left(-b^{N}(w, w)\right)$ is $L^{2}(\Gamma)$-elliptic, i.e. 


$$
\operatorname{Im}\left(-b^{N}(w, w)\right) \geq c_{0}^{\prime}\|w\|_{L^{2}(\Gamma)}^{2} \text { and }\left|b^{N}(w, w)\right| \geq c_{0}\|w\|_{L^{2}(\Gamma)}^{2},
$$

where $\quad c_{0}^{\prime}=\min \left\{\frac{2}{\pi R\left|H_{N}^{(1)}(k R)\right|^{2}}, k\right\}>0, \quad c_{0}=\frac{1}{\sqrt{2}} \min \left\{\min _{|m| \leq N}\left|k H_{|m|}(k R)\right|, k+\frac{1}{2 R}\right\}>0$.

The variational problem corresponding to (7) is: Find $u_{h}^{N} \in H^{1}(\Gamma)$ such that

$$
b^{N}\left(u_{h}^{N}, v_{h}\right)=<g, v_{h}>, \quad \forall v_{h} \in V_{h}(\Gamma) .
$$

Theorem 3. Let $g^{\in} L^{2}(\Gamma)$, then the variational problem (13) has a unique solution $u_{h}^{N} \in L^{2}(\Gamma)$.

Let $g^{\in} L^{2}(\Gamma)$, from Theorem 1, we known that (7) has a unique solution $u^{\in} H^{1}(I)$. The numerical solution $u_{h}^{N} \in V_{h}(\Gamma) \subset H^{1}(\Gamma)$ by (13).

Theorem 4. Let $g^{\in} L^{2}(\Gamma), u$ is the solution of (7). $u_{h}^{N}$ is the solution of (13). Supposed that $u \in H^{p+1}(\Gamma), p \geq 1$, and the finite element interpolation $\Pi: H^{p+1}(\Gamma) \rightarrow V_{h}$ satisfies $\|u-\Pi u\|_{H^{1}(\Gamma)} \leq C_{0} h^{p}|u|_{p+1}$, then

$$
\left\|u-u_{h}^{N}\right\|_{L^{2}(\Gamma)} \leq C_{1} h^{p}|u|_{p+1}+C_{2} R^{N}(u, k, R),
$$

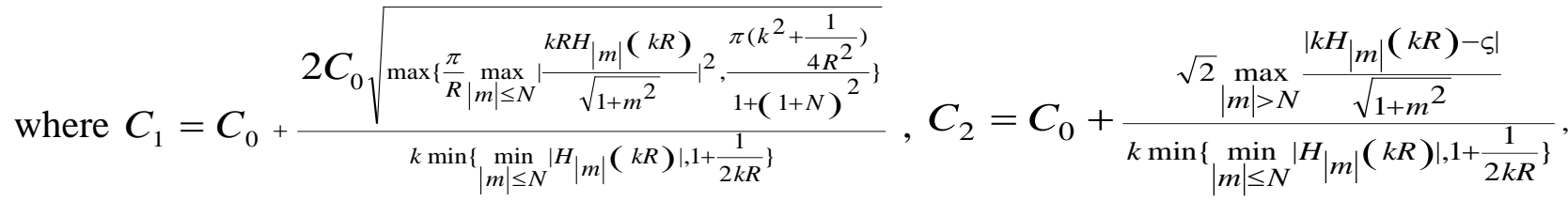
$R^{N}(u, k, R)=\left(\sum_{|m|>N}\left(1+|m|^{2}\right)\left|b_{m}\right|^{2}\right)^{\frac{1}{2}}$ and $b_{m}=\frac{1}{2 \pi} \int_{0}^{2 \pi} u(\theta) e^{-i m \theta} d \theta$.

Proof. $\forall v \in V_{h}$, from the variational problems (7), (12) and (13), we have

$$
b^{N}\left(u_{h}^{N}-v, u_{h}^{N}-v\right)=b^{N}\left(u-v, u_{h}^{N}-v\right)-b^{N}\left(u, u_{h}^{N}-v\right)+a\left(u, u_{h}^{N}-v\right)
$$

Because $u_{h}^{N}-v \in V_{h}$, let $u=\sum_{m=-\infty}^{+\infty} b_{m} e^{i m \theta}, u-v=\sum_{m=-\infty}^{+\infty} c_{m} e^{i m \theta}$ and $u_{h}^{N}-v=\sum_{m=-\infty}^{+\infty} d_{m} e^{i m \theta}$, according to Lemma 4 and 3, we can obtain

$$
\begin{aligned}
\left\|u_{h}^{N}-v\right\|_{L^{2}(\Gamma)}^{2} & \leq c_{0}^{-1}\left|b^{N}\left(u_{h}^{N}-v, u_{h}^{N}-v\right)\right| \\
& =c_{0}^{-1}\left|b^{N}\left(u-v, u_{h}^{N}-v\right)-b^{N}\left(u, u_{h}^{N}-v\right)+a\left(u, u_{h}^{N}-v\right)\right| \\
& =c_{0}^{-1}\left(\left|<L^{N}(u-v), u_{h}^{N}-v>\right|+\left|<\left(K-L^{N}\right) u, u_{h}^{N}-v>\right|\right) \\
& \leq \frac{c_{3}}{c_{0}}\|u-v\|_{H^{1}(\Gamma)}\left\|u_{h}^{N}-v\right\|_{L^{2}(\Gamma)}+\frac{1}{c_{0}} \max _{|m|>N} \frac{\left|k H_{|m|}(k R)-\varsigma\right|}{\sqrt{1+m^{2}}} R^{N}(u, k, R)\left\|u_{h}^{N}-v\right\|_{L^{2}(\Gamma) .}
\end{aligned}
$$

From the triangle inequality, we have

$$
\left\|u-u_{h}^{N}\right\|_{L^{2}(\Gamma)} \leq\|u-v\|_{L^{2}(\Gamma)}+\left\|u_{h}^{N}-v\right\|_{L^{2}(\Gamma)} \leq\left(1+C_{3} C_{0}^{-1}\right)\|u-v\|_{H^{1}(\Gamma)}+C_{2} R^{N}(u, k, R) .
$$

So, we obtain

$$
\left\|u-u_{h}^{N}\right\|_{L^{2}(\Gamma)} \leq\left(1+c_{3} C_{0}^{-1}\right)\|u-\Pi u\|_{H^{1}(\Gamma)}+C_{2} R^{N}(u, k, R) \leq C_{0}\left(1+c_{3} C_{0}^{-1}\right) h^{p}|u|_{p+1}+C_{2} R^{N}(u, k, R) .
$$

\section{The numerical example}

Numerical results of examples are obtained by MNBEM as follows, where

$$
\left\|u-u_{h}^{N}\right\|_{L^{2}(\Gamma)}=\sqrt{\sum_{i=1}^{M}\left(\theta_{i+1}-\theta_{i}\right)\left(u\left(x_{i}, y_{i}\right)-u_{h}^{N}\left(x_{i}, y_{i}\right)\right)^{2}}, \text { Rate }=\frac{\left\|u-u_{h}^{N}\right\|_{L^{2}(\Gamma)}}{\left\|u-u_{\frac{h}{2}}^{N}\right\|_{L^{2}(\Gamma)}}, \quad \text { Order }=\log _{2} \text { Rate. }
$$

Example. Let $\Omega=\left\{(x, y) \mid x^{2}+y^{2}>225\right\}$ and $\Gamma=\partial \Omega$, consider the following equations 


$$
\left\{\begin{array}{l}
\Delta u+k^{2} u=0, \text { in } \Omega, \\
\frac{\partial u}{\partial n}=e^{3 i \theta}+e^{6 i \theta}+e^{10 i \theta}, \text { on } \Gamma,
\end{array}\right.
$$

with the Sommerfeld radiation condition. This problem has an exact solution:

$$
u(r, \theta)=-\frac{H_{3}^{(1)}(k r)}{k H_{3}^{(1)}(15 k)} e^{3 i \theta}-\frac{H_{6}^{(1)}(k r)}{k H_{6}^{(1)}{ }^{\prime}(15 k)} e^{6 i \theta}-\frac{H_{10}^{(1)}(k r)}{k H_{10}^{(1)}{ }^{\prime}(15 k)} e^{10 i \theta}, r \geq 15,0 \leq \theta<2 \pi .
$$

Table 1. When $k=1$, the errors of the MNBEM

\begin{tabular}{ccccccccc}
\hline Error & $M$ & $N=5$ & Rate & $N=6$ & Rate & $N=10$ & Rate & Order \\
\hline$\left\|u-u_{h}^{N}\right\|_{L^{2}(\Gamma)}$ & 16 & 0.8669 & & 1.5745 & & 5.7568 & & \\
& 32 & 0.3628 & 2.3896 & 0.3736 & 4.2145 & 0.4621 & 12.4590 & 3.6391 \\
& 64 & 0.0317 & 11.4351 & 0.0478 & 7.8031 & 0.1162 & 3.9774 & 1.9918 \\
& 128 & 0.0531 & 0.5978 & 0.0513 & 0.9313 & 0.0285 & 4.0724 & 2.0259 \\
& 256 & 0.0732 & 0.7250 & 0.0694 & 0.7385 & 0.0071 & 4.0208 & 2.0075 \\
\hline
\end{tabular}

We find in Table 1 that when $N=10$, the MNBEM converges quadratically as $M \rightarrow \infty$, which verifies that $C_{1}$ is a constant and $R^{N}(u, k, R)=0$ in the error estimate for $N \geq 10$. When $N=5$ and 6 , if $M$ is not large enough, due to the accuracy of finite element is improved by increasing $M$ the error of numerical solutions will decrease with the order of $O\left(M^{-2}\right)$. If $M$ is large, then the first part of the error in Theorem 4 will be very small and the second part will play a main role.

\begin{tabular}{|c|c|c|c|c|}
\hline \multirow[t]{2}{*}{$N$} & \multicolumn{2}{|c|}{$M=120$} & \multicolumn{2}{|c|}{$M=200$} \\
\hline & $\left\|u-u_{h}^{N}\right\|_{L^{\infty}(\Gamma)}$ & $\left\|u-u_{h}^{N}\right\|_{L^{2}(\Gamma)}$ & $\left\|u-u_{h}^{N}\right\|_{L^{\infty}(\Gamma)}$ & $\left\|u-u_{h}^{N}\right\|_{L^{2}(\Gamma)}$ \\
\hline 4 & 0.025830 & 0.049366 & 0.035184 & 0.068933 \\
\hline 5 & 0.025830 & 0.049366 & 0.035184 & 0.068933 \\
\hline 6 & 0.023777 & 0.048106 & 0.027899 & 0.065468 \\
\hline 10 & 0.017457 & 0.032487 & 0.006258 & 0.011637 \\
\hline 20 & 0.017457 & 0.032487 & 0.006258 & 0.011637 \\
\hline 40 & 0.017457 & 0.032487 & 0.006258 & 0.011637 \\
\hline
\end{tabular}

Table 2. When $k=2$, the errors of the MNBEM

We see from Table 2 that the MNBEM is well-posed for any $N$.

\section{Conclusions}

We discuss the MNBEM for exterior Neumann boundary value problems of the Helmholtz equation in $R^{2}$ in this paper. The MNBEM is well-posed for any $N$. Moreover, we show that:

(1) when $N \geq m_{\max }$, the MNBEM is second-order convergent with respect to $h$ or $1 / M$;

(2) when $N<m_{\max }$, the MNBEM is nearly second-order convergent with respect to $h$ when $M$ is not large enough and almost holds on the accuracy when $M$ is larger; where $m_{\max }$ is the highest frequency of the exact solution.

\section{Acknowledgement}

The work is supported by Natural Science Foundation of China (11471019).

\section{References}

[1] K. Feng, D. Yu, Canonical integral equations of elliptic boundary value problems and their numerical solutions. In: (K. Feng, J.L. Lions, eds.) Proc. China-France Symp. on the Finite Element Method (April 1982), pp. 211-252. Beijing: Science Press, 1983.

[2] K. Feng, Differential vs. integral equations and finite vs. infinite elements, Math. Numer. Sin. 2 (1980) 100-195.

[3] R. Li, Natural boundary enement method for the exterior boundary value problems of 
Helmholtz equation (in Chinese), Appl. Math. -J.C.U. 12 (1997) 369-374.

[4] J.B. Keller, D. Givoli, Exact nonreflecting boundary condition, J. Comput. Phys. 82 (1989) 172-192.

[5] M.G. Grote, J.B. Keller, On nonrefleting boundary conditions, J. Comput. Phys. 122 (1995) 231-243.

[6] D. Koyama, Error estimates of the finite element method for the exterior Helmholtz problem with a modified DtN boundary condition, J. Comput. Appl. Math. 232 (2009) 109-121.

[7] D. Koyama, Error estimates of the DtN finite element method for the exterior Helmholtz problem, J. Comput. Appl. Math. 200 (2007) 21-31.

[8] G.C. Hsiao, N. Nigamb, J.E. Pasciak, L. Xu, Error analysis of the DtN-FEM for the scattering problem in acoustics via Fourier analysis, J. Comput. Appl. Math. 235 (2011) 4949-4965.

[9] H. Han,W. Bao, Error estimates for the finite element approximation of problems in unbounded domains, SIAM J. Numer. Anal. 37 (2000) 1101-1119. 